\title{
PW05-02
}

\section{CARDIOVASCULAR RISK IN A SPANISH POPULATION OF BIPOLAR DISORDER PATIENTS: RESULTS FROM THE BIMET} STUDY

\author{
J.M. Montes ${ }^{1}$, E. Vieta ${ }^{2}$, A. González-Pinto ${ }^{3}$, J. Rejas-Gutiérrez ${ }^{4}$, F. Mesa ${ }^{5}$ \\ ${ }^{1}$ Department of Psychiatry, Hospital del Sureste, Madrid, ${ }^{2}$ Department of Psychiatry, Hospital Clínic and IDIBAPS, Neurosciences \\ CIBERSAM, Barcelona, ${ }^{3}$ Department of Psychiatry, Hospital Santiago Apostol and Stanley Research Center, Psychiatry \\ CIBERSAM, Vitoria, ${ }^{4}$ Health Outcomes Research Department, Medical Unit, Pfizer Spain, ${ }^{5}$ Medical Unit, Pfizer Spain, Alcobendas, \\ Madrid, Spain
}

Purpose: To estimate the 10-years coronary heart disease and cardiovascular mortality risks in a Spanish population of bipolar disorder (BD) patients.

Methods: BIMET study is a 12-month, prospective, multicentre, naturalistic study which enrolled type I or II BD patients according to DSM-IV TR criteria. A fasting blood sample was drawn to evaluate glucose, total cholesterol, HDL cholesterol, LDL cholesterol and triglycerides. Weight, height, waist circumference at the umbilicus and blood pressure were also recorded. Framinghan and SCORE functions were used to calculate the 10-year risk for coronary hearth disease or cardiovascular death respectively.

Results: A total of 320 and 417 patients, mean age 46.3 (13.0) years, were included for the Framingham and SCORE calculations respectively. The overall mean (standard deviation) risk for coronary heart disease was $7.3 \%$ (7.8) according to Framingham algorithm, with $6.3 \%$ of patients classified as having a high risk of coronary disease (Framingham higher than $10 \%$ ). The mean overall risk for cardiovascular mortality was 1.2\% (2.4) according to SCORE algorithm, with $6.5 \%$ of patients falling within the high cardiovascular mortality risk segment; SCORE higher than $5 \%$. No statistically significant differences were observed between type I and II patients.

Conclusion: This study reflects a substantial risk for cardiovascular events or mortality in a Spanish cohort of bipolar disorder patients. Results were comparable to those seen in subject with schizophrenia spectrum disorders. Therefore, an effort for controlling all cardiovascular risk factors in bipolar disorder patients should be carried out by clinicians and health decision makers. 